\title{
Visible Contrast Energy Metrics for Detection and Discrimination
}

\author{
Al Ahumada
}

al.ahumada@nasa.gov

Beau Watson

andrew.b.watson@nasa.gov

NASA Ames Research Center

Moffett Field, CA 


\section{Energy Metric for Detection}

- Inputs: Luminance image $=\mathrm{L}(\mathrm{x}, \mathrm{y})$

Pixel area $=\mathrm{dx} d \mathrm{y}$ in $\operatorname{deg}^{2}$,

Duration $=\mathrm{dt}$ in $\mathrm{sec}$

- Compute visible contrast image $=\mathrm{Cv}(\mathrm{x}, \mathrm{y})$

- Visible Energy Metric :

$E v=d x d y d t \sum_{x, y} C v(x, y)^{2} \operatorname{deg}^{2} \sec$ $\mathrm{dBV}=10 \log _{10}\left(\mathrm{Ev} / 10^{-6}\right)$

- Modelfest average threshold $=7 \pm 2 \mathrm{dBV}$ 


\section{Visible Contrast Image}

- Optic Blur : $L o(x, y)=L(x, y){ }^{*} O(x, y)$

- Background Luminance :

$\mathrm{Lb}(\mathrm{x}, \mathrm{y})=\mathrm{a}(\mathrm{dt})\left(\mathrm{Lo}(\mathrm{x}, \mathrm{y}){ }^{*} \mathrm{~B}(\mathrm{x}, \mathrm{y})\right)+(1-\mathrm{a}(\mathrm{dt})) \mathrm{B}_{0}$

- Contrast: $\mathrm{C}(\mathrm{x}, \mathrm{y})=\frac{\mathrm{Lo}(\mathrm{x}, \mathrm{y})-\mathrm{Lb}(\mathrm{x}, \mathrm{y})}{\mathrm{Lb}(\mathrm{x}, \mathrm{y})}$

- Eccentricity Sensitivity :

$$
C v(x, y)=C(x, y) S(x, y)
$$




\section{Parameters}

- Optic Blur : $F(O(x, y))=\exp \left(-f / f_{0}\right)$,

$$
f=\sqrt{ }\left(f_{x}{ }^{2}+f_{y}{ }^{2}\right), f_{0}=12 \mathrm{cpd}
$$

- Background Luminance :

$$
\begin{aligned}
& F(B(x, y))=\exp \left(-\left(f / f_{1}\right)^{2}\right), f_{1}=2 \mathrm{cpd} \\
& a(d t)=\exp \left(-d t / t_{0}\right), t_{0}=0.4 \mathrm{sec}
\end{aligned}
$$

- Eccentricity Sensitivity :

$$
\begin{aligned}
& S(x, y)=1 /\left(1+g\left(1-\exp \left(-r / r_{0}\right)\right)\right) \\
& r=\sqrt{ }\left(x^{2}+y^{2}\right), r_{0}=5.7 \mathrm{deg}, \\
& g=4.1,1 /(1+g)=0.2
\end{aligned}
$$




\section{Metric-Validating Model}

- Visibility Image: Cv(x,y)

- Additive White Noise with 2-sided power spectral density

$\mathrm{N}=\sigma^{2} \mathrm{dx} d \mathrm{y} d \mathrm{t}$,

Each pixel is independently distributed as

Normal with mean 0 and standard deviation $\sigma$

- Ideal Observer detects presence or absence of signal in a two interval forced choice (2IFC) experiment. 


\section{IFC Model Performance}

- Visibility Image: $\mathrm{Cv}(\mathrm{x}, \mathrm{y})$ with visible contrast energy Ev and noise spectral density $\mathrm{N}$

- Distance between observer output distributions divided by their common standard deviation is

$$
d^{\prime}=\sqrt{ }(2 \mathrm{Ev} / \mathrm{N})
$$

- $\operatorname{Prob}($ Correct $)=P c=F z\left(d^{\prime}\right)-0.5$

- Estimated $\mathrm{N}=2 \mathrm{Ev} / \mathrm{d}^{\prime} 2$

- If $\mathrm{Pc}=0.84, \mathrm{~d}^{\prime}=1, \mathrm{~N}=2 \mathrm{Ev}$

- Modelfest : $10 \log _{10}(\mathrm{~N})+60=10 \pm 2 \mathrm{~dB}$ 


\section{Discrimination Model}

- Visibility Images: $\mathrm{Cv}(\mathrm{x}, \mathrm{y}, \mathrm{j}), \mathrm{j}=1, \mathrm{M}$

- Additive White Noise with power spectral density

$\mathrm{N}=\sigma^{2} \mathrm{dx} d \mathrm{~d} d \mathrm{t}$

- Ideal Observer responds $\mathrm{k}$ if image $\mathrm{j}$ is presented and image $\mathrm{k}$ has the smallest squared distance $d(k)$ to the noisy image

$\mathrm{d}(\mathrm{k})=\|\mathrm{Cv}(\mathrm{j})+\mathrm{N}-\mathrm{Cv}(\mathrm{k})\|^{2}$

$\mathrm{d}(\mathrm{k})=\|\mathrm{Cv}(\mathrm{j})+\mathrm{N}\|^{2}+\|\mathrm{Cv}(\mathrm{k})\|^{2}$

$-2(\operatorname{Cv}(\mathrm{j}) \cdot \operatorname{Cv}(\mathrm{k})+\mathrm{N} \cdot \mathrm{Cv}(\mathrm{k}))$ 


\section{Discrimination Model Metric}

- Orthogonal Images: $\mathrm{Cv}(\mathrm{j}) \cdot \mathrm{Cv}(\mathrm{k})=0, \mathrm{j} \neq \mathrm{k}$

- Same energy: $E v(j)=E v$

- Let $d^{\prime}=\sqrt{ }(E v / N)$

$$
P c=\int F(x)^{k-1} f\left(x-d^{\prime}\right) d x,
$$

where $F$ and $f$ are the cumulative and density distribution functions of the standard normal.

- Also $\mathrm{Ev}=\mathrm{dx}$ dy dt $\sum_{\mathrm{j}}\|\mathrm{Cv}(\mathrm{j})-\mathrm{C}\|^{2} /(\mathrm{M}-1)$ where $C=\sum_{j} C v(j) / M$ 


\section{Discrimination Model Performance}

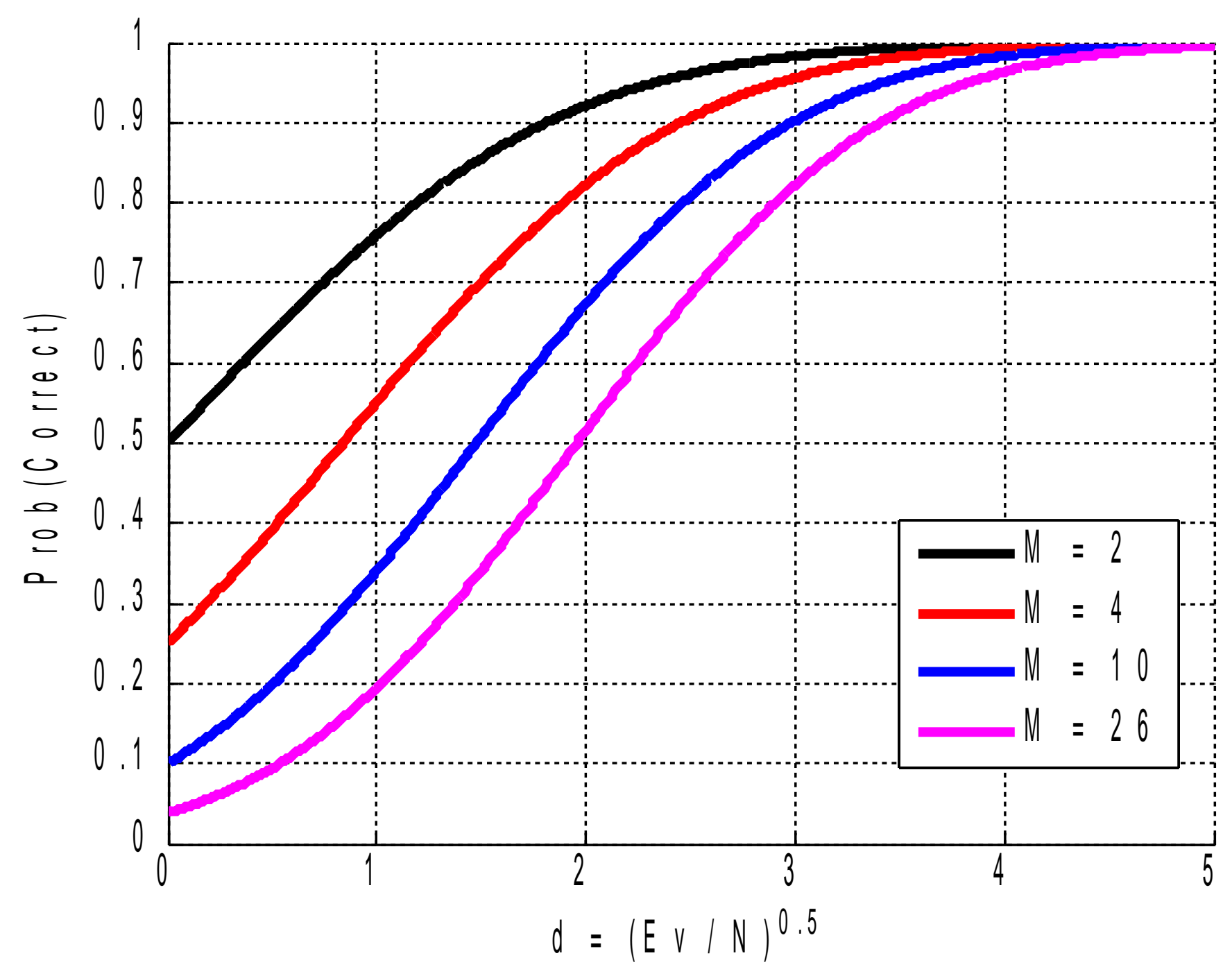




\section{Example: Landolt C}

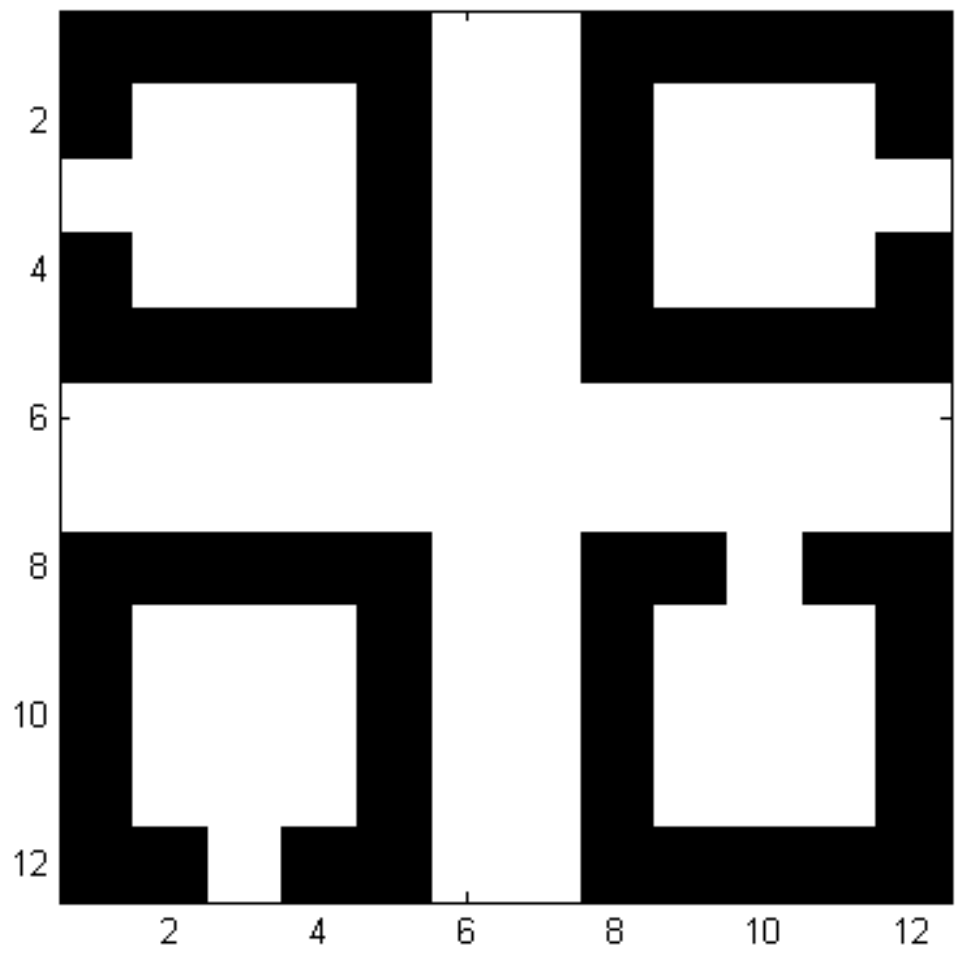

Pedestal invariance of ideal observer allows the orthogonal stimulus model. 


\section{Example: Tumbling E's}

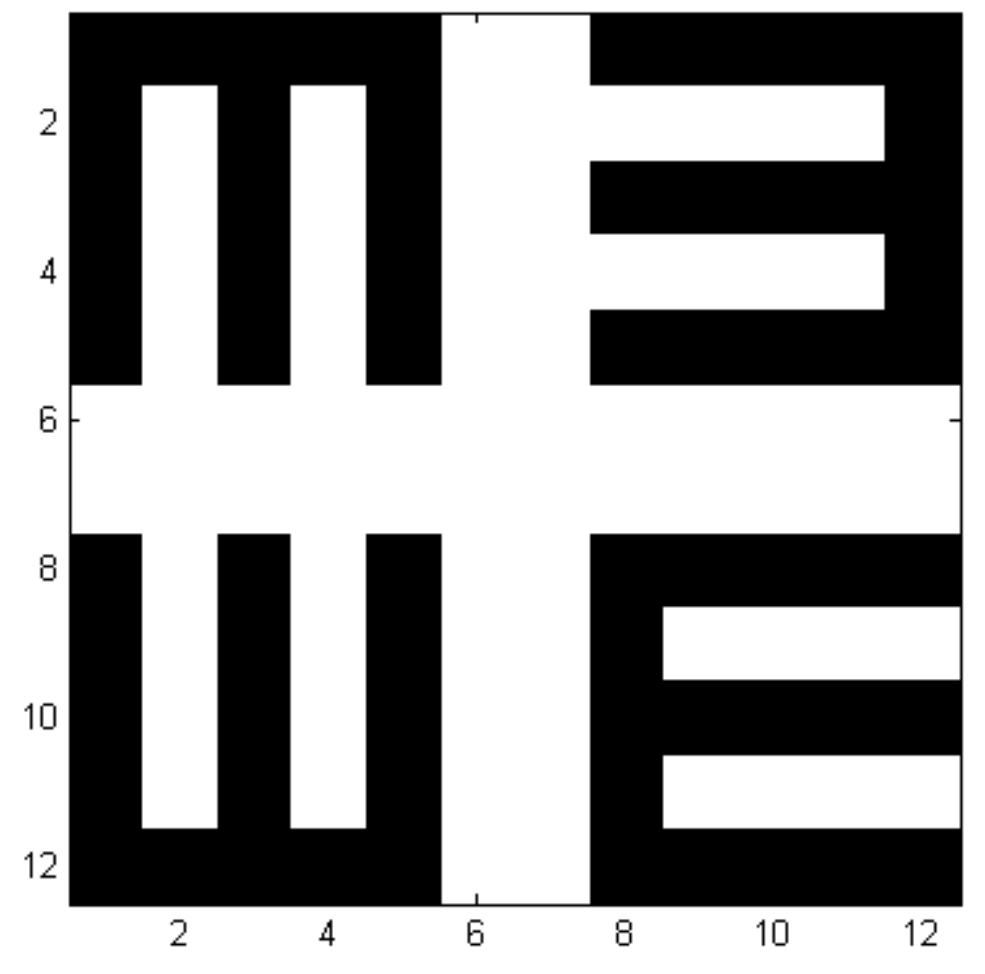

- Model simulation for $n=10000$ trials, $d^{\prime}=1$. $95 \%$ confidence interval for $\mathrm{Pc}=0.538 \pm 0.010$

- Metric prediction Pc $=0.552$ 


\section{Method Considerations}

- When pattern energies are similar, varying the contrast adds little or no uncertainty; varying size or blur contributes significant uncertainty.

- Practice of computing thresholds by averaging reversal endpoints has problems

1) Pc at threshold is not actually known

2) No estimate of the slope at threshold is provided

3) Valuable data is effectively discarded 


\section{Summary}

- Detection metric:

Visible contrast energy

- Approximate Discrimination metric:

Average (M-1) squared distance from each visible contrast pattern to the mean visible contrast pattern

- Model simulation is fast 


\section{Tumbling E Model Matlab Code}

$c=S^{\prime *} S ; \div 4 \times 25$ times $25 \times 4$

$[\mathrm{u}, \mathrm{x}, \mathrm{v}]=\mathrm{svd}(\mathrm{C})$;

$\mathrm{f}=\mathrm{u}^{\star}\left(\mathrm{x} \cdot{ }^{\wedge} 0.5\right)$;

$\mathrm{sn}=$

ones $(n, 4){ }^{*} \mathrm{c}(1,1: 4)+\operatorname{randn}(\mathrm{n}, 4)$ f $^{\prime}$;

$\mathrm{PC}=$ mean (

$\operatorname{sn}(1: n, 1)>\max \left(\operatorname{sn}(1: n, 2: 4)^{\prime}\right) '$

) ; 


\section{Watson \& Ahumada (2005) Metric Elements}

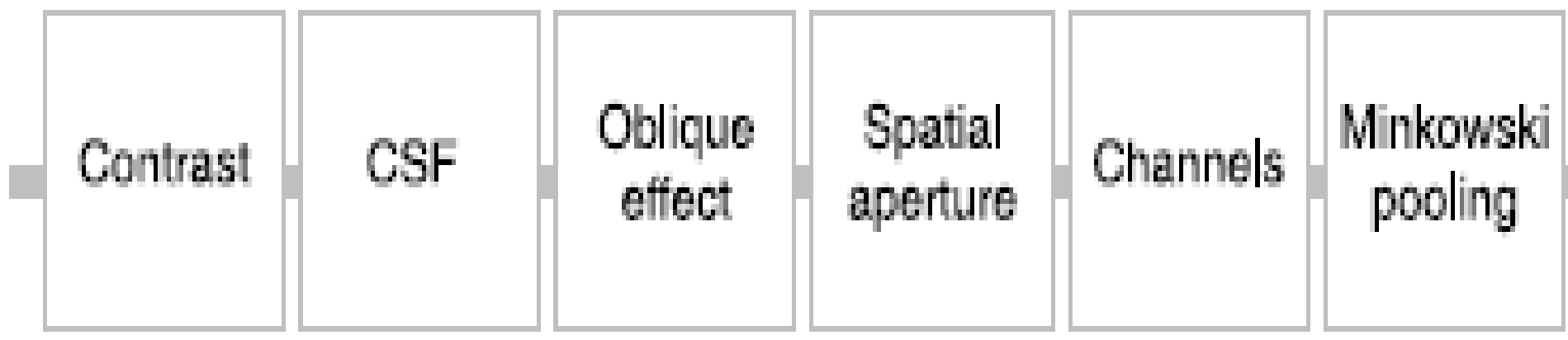

Figure 4. Elements of the component model. 